\title{
Cardiac changes in acute ischemic stroke
}

\author{
Maria Mirabela Manea', Dorin Dragos²,3, Vladimir Moldoveanu', \\ Constantin Popa ${ }^{1,3}$, Sorin Tuta ${ }^{1,3}$ \\ ${ }^{1}$ National Institute of Neurology and Neurovascular Diseases, Bucharest, Romania \\ 2Emergency University Hospital, Bucharest, Romania \\ 3"Carol Davila"University of Medicine and Pharmacy, Bucharest, Romania
}

\begin{abstract}
Purpose: the analysis of the paraclinical features of heart changes in the acute ischemic stroke, especially electrocardiographic (ECG) abnormalities.

Material and methods. We performed a prospective study on 23 patients admitted in our stroke unit with large lesions induced by acute ischemic strokes and no history of cardiovascular disease.

Results. The average age of patients was $72,87+/-11,55$ years. On admission NIHSS score was higher in the cardioembolic stroke patients compared to atherothrombotic stroke patients, the difference persisting after the first seven days. Echocardiography demonstrated a larger left atrium area in cardioembolic compared to atherothrombotic stroke patients. The E-wave deceleration time (a diastolic function parameter) was longer in atherothrombotic compared to cardioembolic stroke. The ECG monitoring and repeated 24h Holter monitoring detected atrial fibrillation (AF) in $52,17 \%$ of the patients and it was associated with a higher in-hospital mortality and stroke severity. No significant correlation was found between the increase in troponin $\mathrm{T}$ levels and AF, or in-hospital mortality.

Discusions. In our study atherothrombotic stroke is associated with a more pronounced tendency to diastolic dysfunction compared to cardioembolic stroke. The percentage of detected AF is higher than expected because of: 1 ) the higher average age of patients, 2) the inclusion of patients with large strokes, and 3) continuous ECG and Holter monitoring for longer periods of time compared to the usual procedure in stroke patients.

Conclusion. The cardiologic monitoring has strong implications for stroke mechanisms and short and long term outcome and prognosis of the patients.
\end{abstract}

Keywords: stroke, diastolic dysfunction, atrial fibrillation

\section{INTRODUCTION}

Cardiogenic embolism accounts for up to $30 \%$ of all ischemic strokes (1). The prevalence of atrial fibrillation (AF) among the patients with ischemic stroke varies between $15 \%$ and $38 \%$ (2), but in some higher risk subgroups it can be detected in more than $50 \%$ of patients (3). Although classical opinion is that $\mathrm{AF}$ is a frequent cause of cerebral embolism, sometimes AF may be the consequence of neurogenic injury (4).

Cerebral (especially insular) injuries may indeed produce neurogenic cardiac arrhythmias, their incidence reaching an early peak in the first 24 hours, but continuing up to 5 days after the stroke onset (5).

Some of the most frequent ischemic ECG abnormalities and arrhythmias in patients with acute stroke are ST segment abnormalities, negative T waves, AF/atrial flutter, sinus tachycardia or bradyarrhythmias (sinus-node dysfunction, $2^{\text {nd }}$ and $3^{\text {rd }}$ degree heart block) (5).

Transthoracic echocardiographic exam may reveal cardiac anomalies associated with an increased risk for embolism: such as cardiac myxoma, cardiomyopathies, anomalies of the mitral valve apparatus, left atrial dilation (which may be at the origin of AF), sustaining that AF is the primary cause of the stroke (6).

Sometimes AF remains one of the undiagnosed causes of embolic stroke. However, perseverant continuous ECG monitoring (using implantable devices of telemetric ECG) for more than one month, increases the probability to detect episodes of paroxysmal AF (7). 


\section{PURPOSE}

The purpose of this small study was the analysis of the paraclinical features of heart changes in the acute ischemic stroke, especially ECG abnormalities in a subset of patients with large ischemic cerebral lesions selected from the stroke unit of our hospital.

\section{SUBJECTS AND METHODS}

We have selected patients with acute $(<24$ hours from onset) ischemic stroke not fulfilling the exclusion criteria: signs of acute or recent (within the last 3 months) myocardial infarction, known AF, cardiac surgery within the last year, active neoplastic disease, and chronic kidney disease (glomerular filtrate rate $<50 \mathrm{ml} / \mathrm{min}$ ), agitation or severe impairment of consciousness (Glasgow coma scale below 8). Between January 2016 and July 2016, 23 of the patients with acute ischemic stroke admitted to the National Institute of Neurology and Neurovascular Diseases - Acute Stroke Unit were enrolled in our study.

Severity of ischemic stroke was assessed using the National Institutes of Health Stroke Scale (NIHSS) at admission and after 7 days and also the modified Rankin scale. All diagnostic procedures and the treatment were in accordance with current guidelines, and an informed consent was obtained from every patient or from his/her family when appropriate.

Diagnostic: Cerebral imaging was performed using computed tomography and, if necessary, magnetic resonance imaging. The continuous monitor ECG for the first 5 days (repeated 24 hours Holter monitoring, but also continuous inpatient ECG monitoring), transthoracic echocardiography and Doppler examination of the cervical and when possible cerebral arteries were also performed.

The TOAST (Trial of Org 10172 in Acute Treatment) classification was used for different stroke subtype (8).

Patients with lacunar stroke, other etiology (using TOAST classification) were not excluded from the study, however, these types of strokes are usually not admitted in our stroke unit (where generally are admitted large strokes, cerebral hemorrhages, candidates for stroke thrombolysis or patients with associated acute heart and coronary diseases). Consequently the study included a subgroup of patients with large cerebral ischemic lesions, with higher mean NIHSS score and associating more vascular risk factors than usual patients. Lacking adequate capacity in stroke unit only a part of all ischemic strokes are treated in the stroke unit, which seems to be common practice also in other referral hospitals (9). Consequently an indirect selection bias was generated, inevitably affecting our sample, but also creating the opportunity to investigate whether the cardiac changes in these more severe strokes have some features distinguishing them from the usual ischemic stroke series encountered in other publications.

Blood tests included cardiac markers (creatinekinase- $\mathrm{MB}$, troponin $\mathrm{T}$, troponin I sampled daily, in the first 5 days). Our laboratory'supper reference limit for serum troponinT level in healthy individuals is $0.03 \mathrm{ng} / \mathrm{mL}$.

Given the little number of patients included in our study we have performed the statistical analysis using Fisher's exact test (by means ofhttp://vassarstats.net/fisher $2 \times 3 . h t m l)$ for discrete variables and Mann-Whitney U Test (by means ofhttp://vassarstats.net/utest.html) for continuous variables (10).

\section{RESULTS}

The average age was $72,87+/-11,55$ years. The proportion of males and females was $52,17 \%$ and $47,83 \%$ respectively.
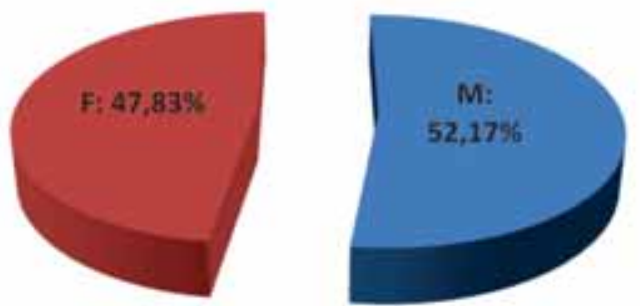

\section{FIGURA 1. Sex distribution}

The TOAST stroke classification: $52,17 \%$ possible cardioembolic (CE), the rest being atherosclerosis related $[47,83 \%$ atherothrombotic (AT)] Fig. 2.

From this last group a small proportion $(8,69 \%)$ had significant stenosis (more than $50 \%$ ) or occlusion of cervical arteries and the related mechanisms of cerebral ischemia being possible atheroembolic (the very few cases of severe stenosis or occlusion were not associated with the watershed lesions suggestive for a hemodynamic infarct).

The mean age was higher in cardioembolic subgroup $(75,3+/-7,96$ years) than in atherosclerosis subgroup (70,2+/-13,5 years), but the difference did not reach a statistical significance due to the small number in Mann-Whitney test. 


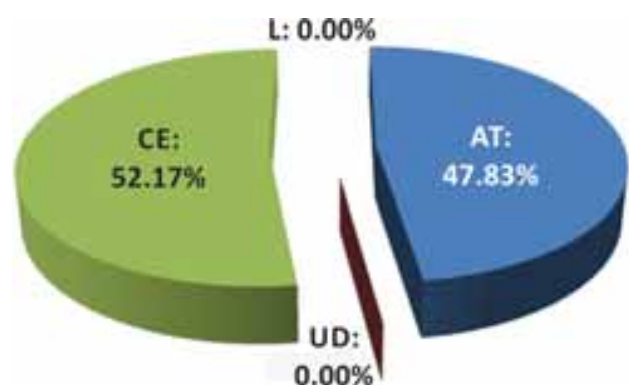

FIGURE 2. TOAST classification (CE = cardioembolic, $A T=$ atherothrombotic, $L=$ lacunar, $U D=$ undetermined)

On admission NIHSS score was higher in the CE stroke patients compared to AT stroke patients (median of 21.5 vs $11, \mathrm{p}=0.02$ by Mann-Whitney test) - Fig. 3, the difference persisting after 7 days, although with insufficient statistical significance. The Rankin score at 7 days was slightly higher (statistically not significant) in CE compared to AT stroke patients.
Left atrium (LA) enlargement is a recognized risk factor for AF (6). There was indeed a tendency towards larger LA areas by echocardiography in CE compared to AT stroke patients $(24,64+/-5,68$ $\mathrm{cm}^{2}$ vs. $19,27+/-5,26 \mathrm{~cm}^{2}$ ), but the degree of overlap between the two sets of values was too big and their medians were too close for statistical significance to be achieved by Mann-Whitney test (Fig.4).

The E-wave deceleration time (EwDT - a diastolic function parameter) was longer in AT compared to CE stroke (a median of $266.5 \mathrm{~ms}$ compared to $168 \mathrm{~ms}, \mathrm{p}=0.03$ by means of by Mann-Whitney test) reflecting a more pronounced tendency to diastolic dysfunction (Fig. 5).

One putative cause for the higher EwDT could be increased thickness of ventricular walls (11), but this was not a valid supposition in our patients as the relation of the posterior wall thickness to EwDT was, if any, rather inverse (a median of 9 in AT
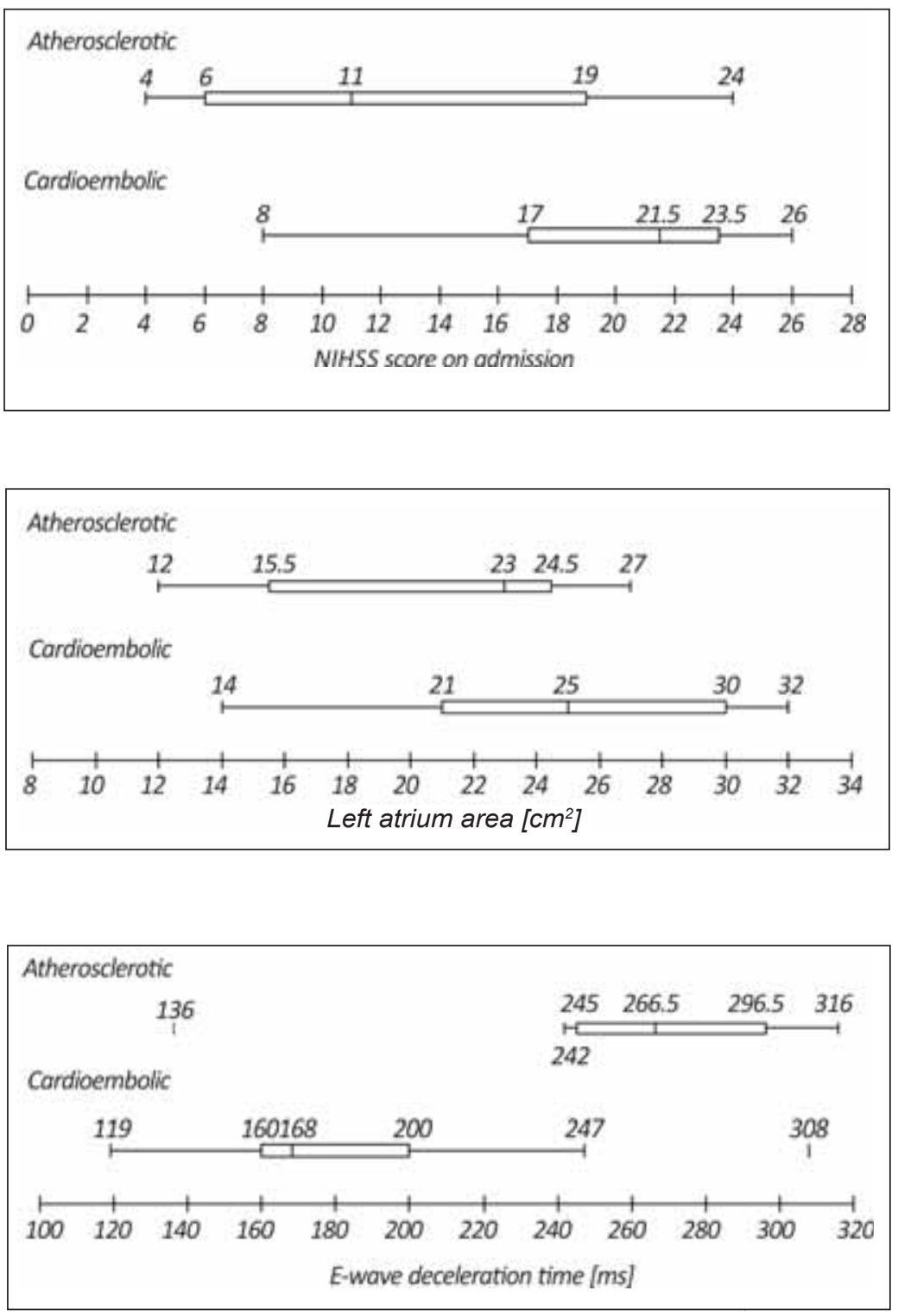

FIGURE 3. NIHSS score on admission correlated with the type of stroke $(p=0.02)$.

FIGURE 4. LA area correlated with the type of stroke $(p=N S)$.

FIGURE 5. E-wave deceleration time correlated with the type of stroke $(p=0.03)$. There are two outliers: 136 for atherosclerotic and 308 for cardioembolic 


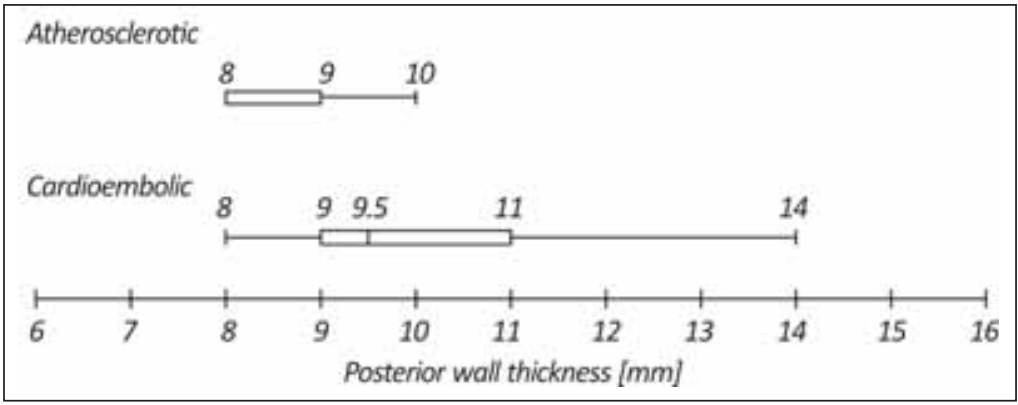

FIGURE 6. Posterior wall thickness correlated with the type of stroke $(p=0.05)$. stroke vs. a median of 10 in CE stroke, $p=0.05$ by Mann-Whitney test) - Fig 6.

Eight out of 22 stroke patients (36\%), where evaluation was possible, had an ejection fraction below $55 \%$.

The continuous ECG monitoring and Holter evaluation: AF was detected in $52.17 \%$ of the patients and it was associated with a higher in-hospital mortality, although not statistically significant. AF has been correlated with stroke severity (subgroup NIHSS analysis) being present in 1 out of 7 $(14.29 \%)$ patients with mild stroke, in 4 out of 7 (57.14\%) patients with moderate stroke, and in 7 out of $9(77.78 \%)$ patients with severe stroke $(\mathrm{p}=$ 0.046 as calculated by Fisher's exact test) - Fig. 7 .

There was a higher tendency to both depression $(0.58+/-0.68 \mathrm{mV}$ vs $0.27+/-0.2 \mathrm{mV})$ and elevation of ST segment $(0.29+/-3 \mathrm{mV}$ vs. $0.2+/-0.16 \mathrm{mV})$ in CE compared to AT strokes, but not statistically significant.

Surprinsigly, in our study, the blood chemistry revealed no correlation between the increase in troponin $\mathrm{T}$ (above $0.03 \mathrm{UI} / \mathrm{L}$ ) and $\mathrm{AF}$, or between the increase in troponin $\mathrm{T}$ and in-hospital mortality, but this could be related to the small number of patients included in this study (type II error).

\section{DISCUSSIONS AND CONCLUSION}

In our study AT stroke was associated with mild diastolic dysfunction, but the underlying mechanism is yet unclear and is probably unrelated to the thickness of the ventricular walls (as explained above). More subtle mechanisms could come into play including relaxation impairment due to ischemia and/or microvascular tone alterations induced by the vegetative disturbances generated by the cerebral injury. Increasing the number of patients included in our study may prove useful in unraveling new unsuspected correlations that may shed some light upon this elusive problem.

The apparently unexpectedly high percentage of detected AF in our study (52.17\%) is not uncommon if we take into account the profile of patients diagnosed with cardioembolic stroke due to $\mathrm{AF}$, with a mean age of 75 years and frequent arterial hypertension and heart failure. In this age subgroup in unselected ischemic stroke cohorts prevalences

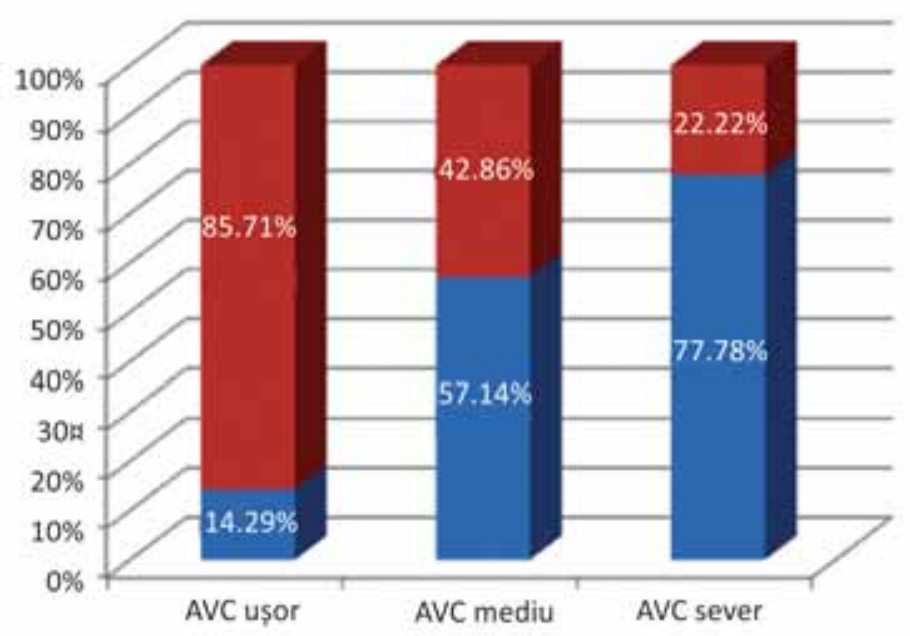


of $\mathrm{AF}$ as high as $38 \%$ were reported $(2,12,13)$. On top on that in other studies (3.14), the combination of arterial hypertension with heart failure (36\% of our patients have had an ejection fraction under $55 \%$ ), was associated with a prevalence of AF in more than $60 \%$ of cases.

In another study (15) more than $60 \%$ of patients with total anterior circulation infarcts were classified as cardioembolic, while the etiology of partial anterior circulation infarcts was either cardioembolic or large-artery atherosclerosis (LAA) in equal ings, bearing in mind that our patients, picked only form the stroke unit have had large, severe ischemic lesions with a median NIHSS score of 21.5).

The Holter monitoring was performed for much longer periods of time (between 3-5 days,) compared to the usual procedure (single 24 hour recording) in stroke patients and direct observation and rewind of the ECG monitoring on electronic devices in the stroke unit, all of these allowing for the detection of a fairly large proportion of paroxysmal AF episodes and of all the cases of persistent AF.

This higher incidence is in line with more recent publications (16) demonstrating that AF prevalence (or rather its detection rate) in acute ischemic stroke and transient ischemic attack has continued to increase.

Our study is also in agreement with other published data, AF being associated with higher in-hospital mortality and increased severity of the ischemic stroke (17). Although all included patients have had relatively high NIHSS scores and relatively large infarcts, those associated with AF had proportions, a result closely mirrored by our find-

significantly higher NIHSS scores and worse prognosis.

The lack of correlation between the troponin $\mathrm{T}$ levels and AF could suggest that AF was more related to preexisting local atrial anomalies rather than to neurogenic heart disease or to acute coronary lesions with de novo arrhythmia associated with acute stroke lesions.

In ordinary neurological wards and highly specialized outpatient units implantable devices for telemetric ECG are not usually available, but some paroxysmal AF episodes may be detected by continuos ECG recording and repeated Holter examination during hospitalization, this kind of monitoring of the patients with acute stroke having strong implications on the detection of stroke mechanisms and on the choice of the appropriate treatment for secondary prevention, but also on the short and long term outcome of the patients. AF remains a too often missed cause of an embolic stroke and is associated with significant risk for recurrent events and severe stroke with higher mortality and invalidity.

In conclusion intensive ECG monitoring and echocardiographic evaluation of severe stroke patients in a stroke unit could diagnose a higher than expected prevalence of AF with significant implications on the prognosis and on the treatment decisions.

\section{Acknowledgements:}

The authors gratefully thank all of the study collaborators

Conflict of interest: none declared Financial support: none declared

\section{REFERENCES}

1. Kishore A., Vail A., Majid A. et al. Detection of Atrial Fibrillation After Ischemic Stroke or Transient Ischemic Attack. A Systematic Review and Meta-Analysis. Stroke. 2014; 45:520-526.

2. Friberg L., Rosenqvist M., Lindgrenet A. et al. High prevalence of atrial fibrillation among patients with ischemic stroke. Stroke. 2014; 45:2599-2605.

3. Haft J.I., Teichholz L.E. High incidence of atrial fibrillation or flutter in stroke patients who have the clinical risk factors for stroke. Journal of Atrial Fibrillation. 2013; 6 :114-119.

4. Kamel H., Peter M., Okin P.M. et al. Atrial Fibrillation and Mechanisms of Stroke. Stroke. 2016; 47:895-900.

5. Kallmünzer B., Breuer L., Kahl N. et al. Serious cardiac arrhythmias after stroke: incidence, time course, and predictors - a systematic, prospective analysis. Stroke. 2012; 43(11):2892-7.

6. Pepi M., Evangelista A., Nihoyannopoulos P. et al. Recommendations for echocardiography use in the diagnosis and management of cardiac sources of embolism. European Journal of Echocardiography. 2010; 11:461-476.
7. Dussault C., Toeg H., Nathan M. et al. Electrocardiographic Monitoring for Detecting Atrial Fibrillation After Ischemic Stroke or logy. 2015; 8:263-269.

8. Adams H.P. Jr., Bendixen B.H., Jaap Kappelle L. et al. Classification of Subtype of Acute Ischemic Stroke. Stroke. 1993; 24:35-41.

9. Stone S. Stroke units - Every patient with a stroke should be treated in a stroke unit. BMJ. 2002; 325:292-3.

10. Lowry R. VassarStats: Web Site for Statistical Computation [http://vassarstats.net], 1998-2017

11. Lalande S., Johnson B.D. Diastolic dysfunction: a link between hypertension abd heart failure. Drugs Today. 2008; 44(7): 503-513.

12. Asberg S., Henriksson K.M., Farahmand B., et al. Ischemic stroke and secondary prevention in clinical practice: a cohort study of 14,529 patients in the Swedish Stroke Register. Stroke. 2010; 41:1338-1342.

13. Björck S., Palaszewski B., Friberg L. et al. Atrial fibrillation, stroke risk, and warfarin therapy revisited: a population-based study.Stroke. 2013; 44:3103-3108. Transient Ischemic Attack. Circulation: Arrhythmia and Electrophysio- 
14. Haft J.I., Teichholz L.E. Clinical heart failure patients with ischemic stroke have a high incidence $(>60 \%)$ of atrial fibrillation or flutter whether systolic function is preserved or depressed. World $J$ Cardiovasc Dis. 2014; 4:455-464.

15. Tei H., Uchiyama S., Koshimizu K. et al. Correlation between symptomatic, radiological and etiological diagnosis in acute ischemic stroke. ActaNeurol Scand. 1999; 99(3):192-5.
16. Otite F.O., Khandelwal P., Chaturvedi S. et al. Increasing atrial fibrillation prevalence in acute ischemic stroke and TIA. Neurology. 2016; 87:2034-2042.

17. Henninger N., Goddeau P.R. Jr, Karmarkar A. et al. Atrial fibrillation is associated with a worse 90 -day outcome than other cardioembolic stroke subtypes. Stroke. 2016; 47:1486-1492. 